\title{
Urogynäkologie / Urologie für die Frau
}

\author{
Sonja Brandner, Cornelia Betschart, Daniel Faltin
}

Dres. med., Ausbildungskommission der Arbeitsgemeinschaft für Urogynäkologie

\begin{abstract}
Am 1.1.2016 trat der neue Schwerpunkt Urogynäkologie / Urologie für die Frau in Kraft. Dieser hat zum Ziel, Kompetenzen im Gebiet der Beckenbodenpathologien und ihrer Folgeerkrankungen zu stärken. Die Arbeitsgemeinschaft für Urogynäkologie der gynécologie suisse SGGG stellt hiermit ihr Nachwuchsförderungsprogramm vor, welches in Zusammenarbeit mit dem jungen Forum der gynécologie suisse SGGG und in Absprache mit der Chefärztekonferenz erarbeitet wurde.
\end{abstract}

\begin{abstract}
Der Bedarf an Gesundheitsleistungen steigt in vielen Bereichen der Medizin, so auch auf dem Gebiet der Beckenbodenerkrankungen. Beckenbodenbeschwerden korrelieren global mit dem Anstieg der Lebenserwartung. Aus Untersuchungen der USA geht hervor, dass in den nächsten 30 Jahren der Bedarf an urogynäkologischen Operationen um 45\% ansteigen wird [1]. Diese Zahlen dürften ähnlich sein für die Schweiz, wo hohe sozioökonomische Verhältnisse eine gute Lebensqualität bis ins betagte Alter ermöglichen. Den steigenden Ansprüchen von Frauen jeden Alters an Erhalt von Lebensqualität, Mobilität und ganzheitlicher Gesundheit gilt es, gerecht zu werden. Diese noch nie zuvor dagewesenen Veränderungen tragen zur Erhöhung des Bewusstseins für Beckenbodenerkrankungen und zur Zunahme des Bedarfs an profund ausgebildeten Akteuren in verschiedenen Berufsgruppen bei.
\end{abstract}

\section{Urogynécologie / Urologie de la femme}

La formation approfondie en Urogynécologie / Urologie de la femme est reconnue depuis le premier janvier 2016. Les détails se trouvent sur le site de la FMH [2].

L'association pour l'Urogynécologie de gynécologie suisse (AUG, urogyn.ch) présente un programme d'encouragement à la relève, préparé en collaboration avec le Forum des jeunes gynécologues de gynécologie suisse et la Conférence des médecins-chef de service. «Urogyn-Akademie» est destinée à de jeunes gynécologues ou urologues en formation opératoire motivés.

Ce programme est composé de journées de formation théoriques et de stages de découverte auprès d'experts en urogynécologie (consultations, examens complémentaires, urodynamique, périnéosonographie, traitements conservateurs et chirurgicaux) ainsi que dans les spécialités impliquées dans l'approche multidisciplinaire des pathologies du plancher pelvien (proctologie, urologie, neuro-urologie, physiothérapie).

L'offre complète est détaillée sur le site (http://urogyn.ch/l-academie-urogyn/jeunes-talents). L'AUG délivre un certificat de participation. Les membres de I'AUG sont à disposition des candidats pour planifier leur formation approfondie et leur projet de carrière en Suisse et à l'étranger.
Mit der Einführung des neuen Schwerpunkttitels Urogynäkologie / Urologie für die Frau per 1.1.2016 konnte die Arbeitsgemeinschaft für Urogynäkologie (AUG) der gynécologie suisse SGGG in Zusammenarbeit mit der Fachgesellschaft für Urologie den Grundstein für eine strukturierte und Qualität sichernde Weiterbildung legen. Die Anforderungen für den Schwerpunkt sind auf der Homepage der FMH zu finden [2].

Nun gilt es, interessierte und engagierte Nachwuchskräfte, nach abgeschlossenem Facharzt für Urologie oder Facharzt für Gynäkologie und Geburtshilfe mit dem Schwerpunkt interventionelle Gynäkologie und Geburtshilfe, für dieses interessante, vielseitige und zukunftsorientierte Fach zu gewinnen und auszubilden. Unter dem Überbegriff «Urogyn-Akademie» soll ein Angebot an interdisziplinären und interprofessionellen Hospitationen und Kursen geschaffen werden, das potentiellen Nachwuchsleuten Einblicke in das vielseitige und spannende Fach bietet und gleichzeitig den Zugang und die Vernetzung mit diversen erfahrenen Spezialistinnen und Spezialisten im Bereich ermöglicht.

Start des neuen Nachwuchsprogrammes (UrogynBasis-Training) mit einer Pilotphase von 1 Jahr ist auf Frühjahr 2016 vorgesehen. Ein umfangreiches Angebot an Hospitationsmöglichkeiten und Kursen in verschiedenen fachärztlichen und paramedizinischen Bereichen wird erfahrenen Assistenzärztinnen und jungen Oberärztinnen auf der Homepage der AUG (http://uro gyn.ch/de/urogyn-akademie/nachwuchsfoerderung) angeboten. Ziel ist es, Einblicke in die Diversität des Faches Urogynäkologie zu bieten, Kontakte zu knüpfen und interessierte, geeignete Nachwuchskräfte in den verschiedenen Landesregionen gezielt zu unterstützen 


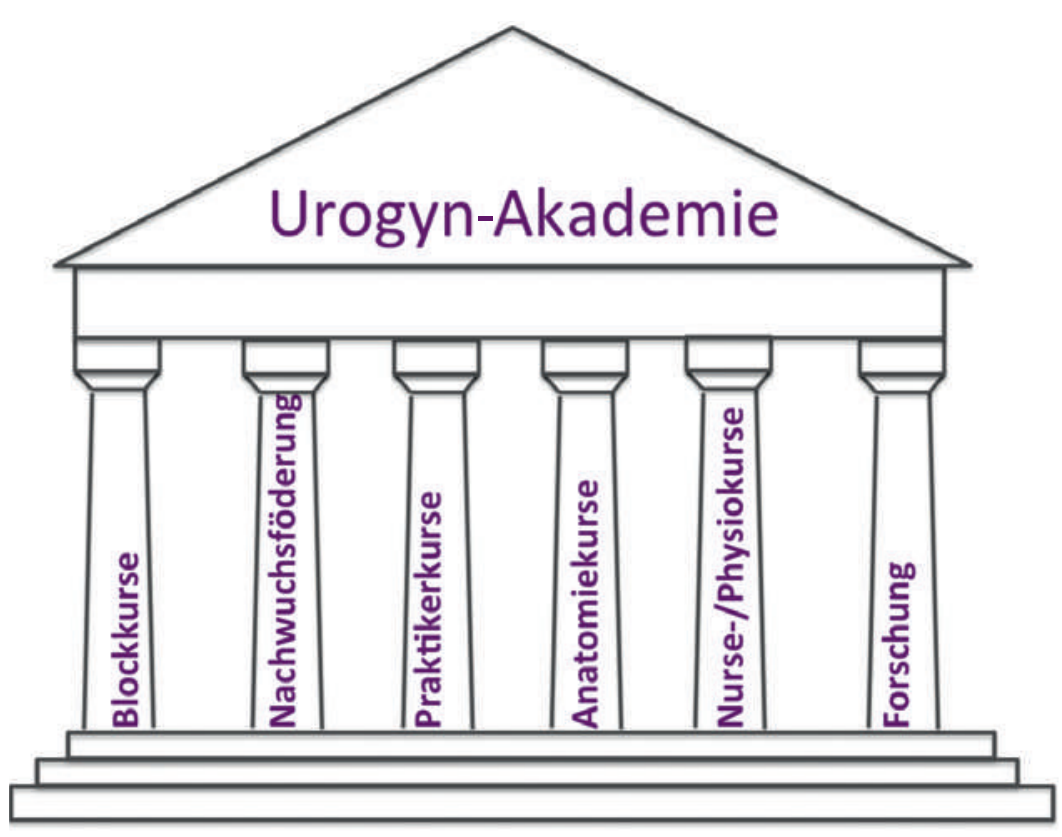

Die «Urogyn-Akademie»: Unter diesem Titel soll ein Angebot an interdisziplinären und interprofessionellen Hospitationen und -kursen geschaffen werden. Bild: Cornelia Betschart

Korrespondenz: Dr. med. Sonja Brandner Monbijoustrasse 22 CH-3011 Bern sonja.brandner[at]hin.ch und in die Weiterbildung aufzunehmen. Die Hospitationstage bieten Einblicke in diagnostische Bereiche (Urodynamik, Perinealultraschall, ...), sowie konservative und operative Therapien. Auch haben Vertreter verschiedener verwandter Bereiche, z.B. Proktologie, Neurourologie, Physiotherapie oder Kontinenzsprechstunden aus den Netzwerken der Beckenbodenzentren ihre Türen zu Hospitationen geöffnet.

Unkompliziert können Interessierte direkt mit den Anbietenden Kontakt aufnehmen und Termine für die Hospitationen vereinbaren. Die Arbeitsgemeinschaft für Urogynäkologie der gynécologie suisse SGGG stellt eine Bestätigung für besuchte Hospitationen aus. Die Mitglieder der AUG unterstützen interessierte und geeignete Kandidatinnen und Kandidaten für den Schwerpunkt auch in der zielorientierten Planung von Weiterbildungsschritten, Auslandsaufenthalten und in der gezielten Karriereplanung. Nach 1 Jahr erfolgt eine Evaluation des Programmes unter Einbezug des Jungen Forums der gynécologie suisse, welches das Programm sehr begrüsst hat und als die hauptsächliche Zielgruppe seine Bedürfnisse einbringen soll. Um einer Spezialisierung im Sinne einer Fragmentierung oder Zersplitterung der bisherigen FMHFacharzttitel vorzubeugen [3], soll die Patientinnenversorgung sinnvoll erweitert und im Verlauf ein umfassendes, niederschwelliges Angebot an Weiterbildungs- möglichkeiten für Niedergelassene und Paramediziner (Pflegefachfrauen in Uro- und Kontinenzpflege, Physiotherapeutinnen für Beckenbodenrehabilitation) aufgebaut werden, was die Versorgungskompetenz nachhaltig und integrativ gewährleisten soll.

Durch direkten Kontakt mit Kolleginnen und Kollegen, welche bereits vertieft in diesem Gebiet arbeiten, soll ein Netzwerk diverser Kompetenzen entstehen mit Fokus auf interdisziplinäre und interprofessionelle Ansätze.

\section{Ausblick}

Der Bedarf an kompetenten Fachleuten zur Behandlung von Beckenbodenerkrankungen wird in Zukunft kaum alleine durch ärztliches Personal abgedeckt werden können. Neben kompetenten Physiotherapeutinnen wird es auch im Pflegebereich ein Angebot der vertieften Weiterbildung in Beratung und Behandlung von Inkontinenz und Prolapserkrankungen brauchen. Im Rahmen der Urogyn-Akademie unterstützt und hilft die AUG in der Weiterentwicklung entsprechender Kursangebote.

\section{Struktur "Urogyn-Basis-Training" \\ zur Nachwuchsförderung}

Fortbildungstage ( 2 theoretische Tage):

z.B. Jahreskongress der gynécologie suisse, UrogynäkologieSymposien Aarau/Frauenfeld/Zürich, Romandie, Internationale Urogynäkologische Kongresse, Blockkurse Urogynäkologie Aarau, Dammrisskurs, EUGA/MPIS/ICS/IUGA, ...

Hospitationstage (4 praktische Tage):

in verschiedenen Fachgebieten (http://urogyn.ch/images/hospitationsplaetze.pdf): Urodynamik, konservative Therapien (Pessare, Instillationen, Physiotherapie), operative Therapien, Bildgebung/Perinealultraschall, Neurourologie, Proktologie, Sexualtherapie, Physiotherapie.

Zertifikat: AUG-Diplom für Basis-Urogynäkologie-Training. Wird nach Nachweis der obigen Hospitations- und Fortbildungstage ausgehändigt (contact[at]urogyn.ch). Das BasisUrogynäkologie-Diplom ist nicht zwingend für die Anmeldung zur Schwerpunktprüfung.

\section{Literatur}

1 Luber KM, Boero S, Choe JY. The demographics of pelvic floor disorders: current observations and future projections. Am J Obstet Gynecol. 2001;184(7):1496-501.

2 http://www.fmh.ch/bildung-siwf/fachgebiete/facharzttitelund-schwerpunkte/gynaekologie-geburtshilfe.html

3 Bauer W. Spezialisierung und Fragmentierung sind zweierlei. Schweiz Ärztezeitung. 2015;96(50-51):1833. 